\title{
Field Electromagnetic
} Strength Variability Measurement and Adaptive Prognostic Approximation with Weighed Least Regression Approach in Ultra-high Radio

\author{
Divine O. Ojuh \\ Department of Physical Sciences, Faculty of Bioloical and Physical Science, Benson Idahosa University, Benin City, \\ Edo State \\ E-mail: dojuh@biu.edu.ng

\section{Joseph Isabona} \\ Department of Physics, Faculty of Science, Federal University Lokoja, PMB. 1154, Lokoja, Kogi State \\ E-mail: josabone@yahoo.com, joseph.isabona@fulkoja.edu.ng
}

Received: 19 March 2021; Accepted: 10 May 2021; Published: 08 August 2021

\begin{abstract}
Propagated electromagnetic signal over the cellular radio communication channels and interfaces are usually highly stochastic and complex with unequal noise variation pattern. This is due to multipath nature of the propagation channels and diverse radio propagation mechanisms that impact the signal strength at the receiver en-route the transmitter, and verse versa. This also makes measurement, predictive modeling and estimation based analysis of such signal very challenging and complex as well. One important and popular parametric modelling and estimation technique in mathematics and engineering science, especially for signal processing applications is the least square regression (LSR). The dominance use and popularity of the LSR approach may be attributed to its simplified supporting theory, relatively fast application procedure and ubiquitous application packages. However, LSR is known to be very sensitive to outliers and unusual stochastic signal data. In this work, we propose the application of weighted least square regression method for enhanced propagation practical field strength estimation modelling over cellular radio communication networks interface. The signal data was collected from a commercial LTE networks service provider. Also, we provide statistical computational analyses to compare the resultant estimation outcome of the weighted least square and the standard least approach. From the result, it is found that the WLSR approach is reliably better the most popular standard least square method. The significance and academic of value of this paper is that our proposed and implemented WLSR method can used as replacement of the standard LSR approach for robust mobile signal processing of future communication system networks.
\end{abstract}

Index Terms: Electric field strength, outliers, estimation accuracy, highly stochastic, heteroskedastic, Weighted, least square regression.

\section{Introduction}

Enhanced field strength estimation-based modelling are useful tools for proper design, simulation and analysis of present and future communication systems. Generally, propagated electromagnetic signal over the cellular radio communication channels and interfaces are usually highly stochastic and complex with unequal noise variance pattern [1]. This is due to multipath nature of the propagation channels, local terrain features and other diverse radio propagation mechanisms that impact the signal strength at the receiver en-route the transmitter, and verse versa [2]. This also makes measurement, predictive modeling and estimation based analysis of such signal very challenging and complex as well. Having a detailed practical knowledge of spatial radio frequency signal variations over cellular network channels and interface is vital in opting for the best adaptive and modulation schemes.

One important and popular parametric modelling and estimation technique in mathematics and engineering science, especially for signal processing applications is the least square regression (LSR). The dominance use and popularity of the LSR approach may be attributed to its simplified supporting theory, relatively fast application procedure and 
ubiquitous application packages. However, one major problem with LSR method is that it is very sensitive to outliers and unusual stochastic signal data.

Our first objective is this paper is to examine the level of spatial electric field variability over LTE cellular network interface in the ultra-high frequency band based on realistic practical measurement. Our second objective is to propose and apply robust weighted least square regression method for enhanced propagation practical field strength estimation modelling over cellular radio communication networks interface.

\section{Literature Review}

A well-known and dominant field strength modelling and estimation technique is literature is the least square regression (LSR). It has been largely applied as a powerful modelling based estimation tool in data mining [3], nuclear science [4, 5], linear system modeling [5], system identification [6], medical imaging [7], and so forth. Particularly, in the domain of signal processing, LSR approach has been robustly used for signal attenuation estimation modelling and propagation loss analysis [8-16]. The dominance use and popularity of the LSR approach may be attributed to its simplified underneath theory and application procedure. Also, LSR application packages are well developed, well documented and very much available. Furthermore, LSR based modeling and computations are relatively fast [12, 13], and optimal in numerous Gaussian situations [10]. However, LSR is known to be very sensitive to extreme observations (i.e. outliers) and unusual stochastic observations [17, 18]. Also, for many non-Gaussian situations, the LSR is far fetch from providing optimal solutions, particularly when the errors trail longer tails type of distributions [19, 20].

In this work, we propose the application of weighted least square regression method for enhanced propagation practical field strength estimation modelling over cellular radio communication networks interface. The signal data was collected from a commercial LTE networks service provider. Also, we provide statistical computational analyses to compare the resultant estimation outcome of the weighted least square and the standard LSR approach.

\section{Methodology}

This work explore realistic practical based technique to study and reliably model electric field strength variability propagated over over LTE cellular network interface in the ultra-high frequency band using robust weighted least square regression. This starts by conducting a detailed field strength drive test measurements around two operational eNodeB transceiver sites belonging to a commercial LTE system networks provider. This is followed by developing a robust weighted least square regression method to model and reliably estimate the measured field strength compared to the standard LSR approach. To measure the estimation accuracy of the proposed WLSR method, we explore six statistical metrics, all which are defined in equations (22) to (26)..

\subsection{Experimental Design and Data Collection}

The electric field strength (EFS) data sample used in this work was obtained from measured LTE radio signal strength data acquired from two operational eNodeB transceiver sites belonging to MTN cellular networks, using Green City Port Harcourt as a case study. The eNodeBs of heights 30 and $32 \mathrm{~m}$, transmits at $2600 \mathrm{MHz}$ frequency. By means of an intensive drive test technique combined with professional Test Mobile System, (TEMS) investigation tools, the signal data was collection was conducted along key pre-identified routes round the two eNodeB sites. The tools, which were connected together before performing the drive test includes the network scanner, a TEMS empowered HP Laptop and mobile phones, dongle, GPS, power inverter and connecting cables.

The EFS is related to LTE signal strength, $\mathrm{P}_{\text {rev }}$ by [21]

$$
\begin{aligned}
& E F S=\left(\frac{P_{e v r}}{\eta} \cdot \frac{G_{a} \lambda^{2}}{4 \pi}\right)^{0.5} \\
& E F S=\left(\frac{P_{e v r}}{480} \cdot \frac{G_{a} \lambda^{2}}{4 \pi}\right)^{0.5}
\end{aligned}
$$

In $d B \mu V / m$, equation (2) is simplified to yield:

$$
E F S=(d B \mu V / m)=P_{r e v}(d B m)+\text { Const }
$$

where 


$$
\text { Const }=20 \log _{10} f(M H z)+77.2(d B)-G_{a}(d B)
$$

\subsection{Weighted Least Square Regression}

The weighted least square regression (WLSR) is a unique parametric regression estimation technique in which the observations are weighted in proportionality to the reciprocal of the estimation error variance for the observations in order to overawe the problem of non-constant variance.

Given a linear model of equation (5) that can employed to estimate and train a set of $y_{i}$ measured data with $y_{1}, y_{2}, \ldots \ldots, y_{N}$ measurement observations (values), that is:

$$
\begin{aligned}
L(x) & =L_{o} x_{o}+L_{1} x_{1} \\
& =\sum_{i=0}^{N-1} L_{i} x_{i}
\end{aligned}
$$

where,

$N=$ no. of training set

$n=$ no. of estimation parameters,

$L_{0}, L_{1}=$ the parameter to be estimated by means of the proposed weighted least square regression. $x_{i}=$ output sequence

$y_{i}=$ measurement values which represent the input sequence

By means of weighted least square regression, the parameters, $L_{0}, L_{1}$ can be estimated by minimizing the square of the weighted residual between the actual measurement output target y and the estimating model value $L\left(x_{i}\right)$ for all $N$ training set with weight, that is:

$$
\operatorname{MSE}(L)=\min \frac{1}{N} \sum_{i=0}^{N} w_{i}\left[L\left(x_{i}\right)-y_{i}\right]^{2}
$$

where $w_{i}$ indicates the weight corresponding to $N$ training set

In Matrix algebra, equation (6) can written as:

$$
\sum_{i=0}^{N-1} w_{i}\left[L\left(x_{i}\right)-y_{i}\right]^{2}=(X L-Y)^{T} W(X L-Y)
$$

where

$$
\begin{aligned}
Y & =\left[\begin{array}{c}
y_{1} \\
y_{2} \\
\vdots \\
y_{t}
\end{array}\right], \text { indicate the measurement observation values } \\
W & =\left[\begin{array}{ccc}
w_{1} & 0 & 0 \\
0 & 0 & 0 \\
\vdots & \vdots & \vdots \\
0 & 0 & w_{t}
\end{array}\right] \text {, the weighted diagonal matrix } \\
X & =\left[\begin{array}{ccc}
1 & \ldots & x_{11} \\
1 & \ldots & x_{21} \\
\vdots & \vdots \\
1 & \ldots & x_{N 1}
\end{array}\right], \text { the input sequence } \\
L & =\left[\begin{array}{cc}
L_{0} \\
L_{1}
\end{array}\right], \text { is the parametric estimation vector }
\end{aligned}
$$

Also, the cost function can be articulated as:

$$
J(L)=\frac{1}{2} \sum_{i=0}^{N-1} w_{i}\left[L\left(x_{i}\right)-y_{i}\right]^{2}=\frac{1}{2}(X L-Y)^{T} W(X L-Y)
$$

To determine estimation parameters, $L_{0}, L_{1}$ which minimize the $J(L)$, we have: 


$$
\begin{gathered}
\frac{\partial}{\partial L} J(L)=\frac{1}{2} \frac{\partial}{\partial L}(X L-Y)^{T} W(X L-Y) \\
=\frac{1}{2} \frac{\partial}{\partial L}\left(L^{T} X^{T} W X L-L^{T} X^{T} W Y-Y^{T} W X L+Y^{T} X^{L} W Y\right) \\
=\left(X^{T} W X L-X^{T} W Y\right)
\end{gathered}
$$

Setting $\frac{\partial}{\partial L} J(L)$ gives

$$
\left(X^{T} W X L-X^{T} W Y\right)=0
$$

The expression in equation (9) leads us to WLSR solution:

$$
L=X^{T} W X-\left(X^{T} W Y\right)^{-1}
$$

For $\mathrm{W}=1$, the expressions in equation (8) to (13) turns to:

$$
\sum_{i=0}^{N-1}\left[L\left(x_{i}\right)-y_{i}\right]^{2}=(X L-Y)^{T}(X L-Y)
$$

Also, the cost function can be articulated as:

$$
J(L)=\frac{1}{2} \sum_{i=0}^{N-1}\left[L\left(x_{i}\right)-y_{i}\right]^{2}=\frac{1}{2}(X L-Y)^{T}(X L-Y)
$$

To determine estimation parameters, $L_{0}, L_{1}$ which minimize the $J(L)$, we have:

$$
\begin{gathered}
\frac{\partial}{\partial L} J(L)=\frac{1}{2} \frac{\partial}{\partial L}(X L-Y)^{T}(X L-Y) \\
=\frac{1}{2} \frac{\partial}{\partial L}\left(L^{T} X^{T} X L-L^{T} X^{T} Y-Y^{T} X L+Y^{T} X^{T} Y\right) \\
=\left(X^{T} X L-X^{T} Y\right)
\end{gathered}
$$

Setting $\frac{\partial}{\partial L} J(L)$ gives

$$
\left(X^{T} X L-X^{T} Y\right)=0
$$

The un-weighted expression in equation (20) defines standard (convectional) parametric estimation approach known as least square regression; that is:

$$
L=X^{T} X\left(X^{T} Y\right)^{-1}
$$

\subsection{Measuring Estimation Accuracy}

To measure the estimation accuracy of WLSR, we explore five statistical metrics. They are mean square error (MSE), root mean square error (RMSE), Mean absolute error (MAE), standard deviation error (STD) and coefficient of determination $\left(\mathrm{R}^{2}\right)$. The five metrics can be defined as: 


$$
\begin{gathered}
\operatorname{MSE}_{\text {WLSR }}=\frac{1}{N} \sum_{i=0}^{N} w_{i}\left[L\left(x_{i}\right)-y_{i}\right]^{2} \\
R M S E_{\text {WLSR }}=\left\{\frac{1}{N} \sum_{i=0}^{N} w_{i}\left[L\left(x_{i}\right)-y_{i}\right]^{2}\right\}^{1 / 2} \\
M A E_{W L S R}=\frac{\sum_{i=1}^{N}\left|w_{i}\left(L\left(x_{i}\right)-y_{i}\right)\right|}{N} \\
S_{T} D_{W L S R}=\left(R M S E_{W L S R}\right)^{2}-\left(\left(M A E_{W L S R}\right)\right)^{0.5} \\
R^{2}=1-\frac{\sum_{i=1}^{N}\left(w_{i}\left(L\left(x_{i}\right)-y_{i}\right)\right)^{2}}{\sum_{i=1}^{N}\left(w_{i}\left(L\left(x_{i}\right)-\operatorname{mean}\left(y_{i}\right)\right)\right)^{2}}
\end{gathered}
$$

\section{Results and Discussion}

First in this section, we provide and discourse the levels of measured field signals across the locations of study. This is followed by presenting the graphical results attained using the proposed weighted least square regression (WLSR) algorithms in adaptive modelling and estimation of the measured field signals. As mentioned earlier, we also explored five statistical metrics such as (MSE), root mean square error (RMSE), Mean absolute error (MAE), standard deviation error (STD) and coefficient of determination $\left(\mathrm{R}^{2}\right)$ to measure and quantify the level of estimation accuracy attained with the proposed WLSR over the standard linear least square approach. All the computer coding, computations and graphics employed to achieve the objective of study was carried using the MATLAB 2018a software platform.

Shown in Figs. 1 to 8 are graphical representations of the electric field strength (EFS) variations and the estimation accuracies made using the proposed weighted least square regression (WLSR) approach against the standard least regression (LSR) approach. A decrease in field strength levels around all measurement eNodeB sites can be observed as a function of measurement distance at the user equipment device. For example, the a $2 d B \mu V / m$ rate of field strength variations is recorded at each measurement distance in site 1, which is about 1.5 times greater than the ones observed in site 2. Having this type of detailed practical knowledge of spatial radio frequency signal variations over cellular network channels and interface is vital in opting for the best adaptive and modulation schemes. The high variations observed in all the measured field strength signals can be ascribed to stochastic multipath nature of the propagation channels and diverse radio propagation mechanisms that impact the signal strength at the receiver en-route the transmitter, and verse versa. Similar reports on varied propagated signals is also contained in [22, 23], but for television signals at $75.25 \mathrm{MHz}$ and $631.25 \mathrm{MHz}$, respectively.

Particularly, in terms of mean absolute error (MAE) as shown in each figure, the results shows that the WLSR based estimation accuracy improves as the EFS data sample sizes, $\mathrm{N}$ increases. This WLSR enviable robust performance can be attributed to its weighted factor which enable to cater for any form of data sample sizes of kinds and usual data values. This is unlike the LSR based estimation degrades with increasing data sample sizes, thus showing that the LSR is negatively sensitive to usual stochastic signal data with outliers. Detailed comparative estimation accuracies made using other statistical metrics like RMSE, PE, STD and $\mathrm{R}^{2}$ are provided in Table 1. From the table, while the 

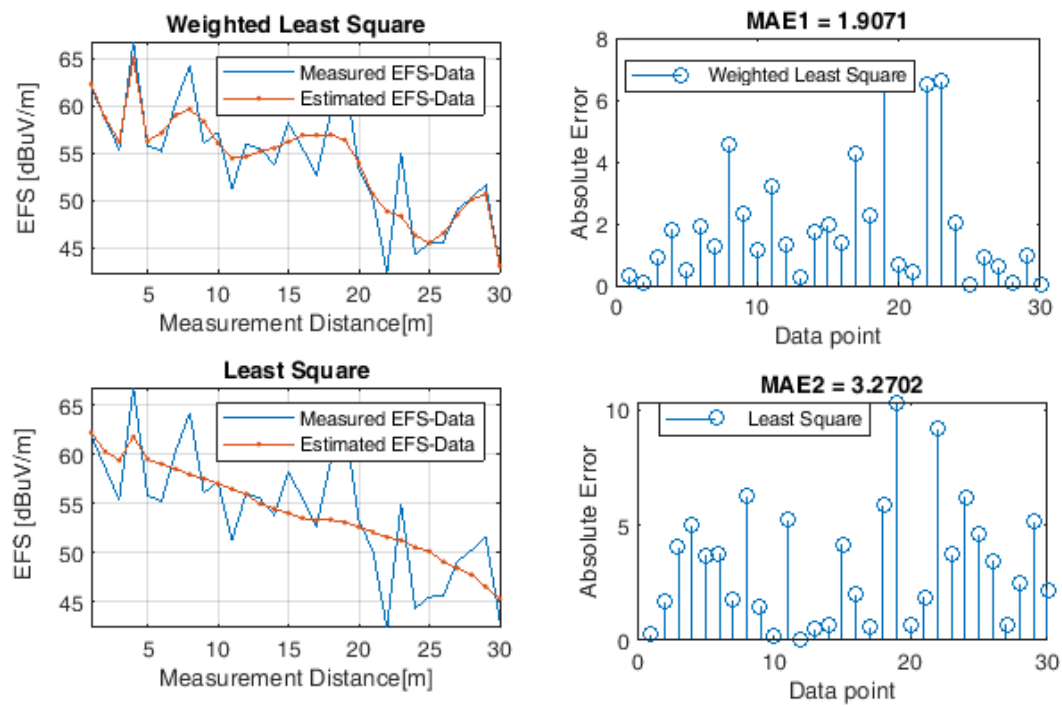

Fig.1. EFS estimation accuracies achieved with WLRS and LSR in site 1 for $\mathrm{N}=30$
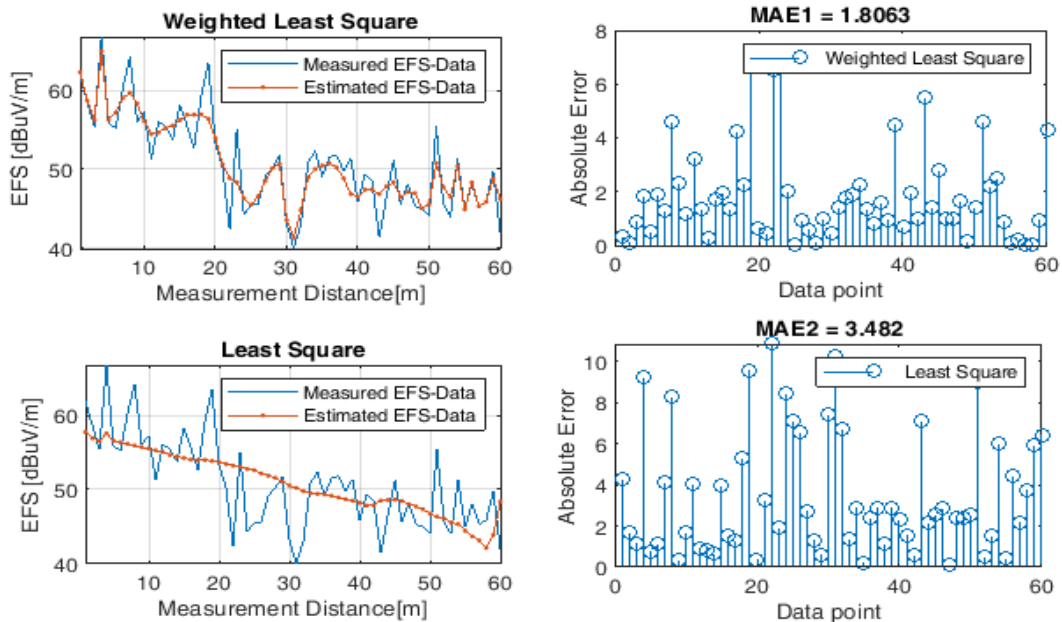

Fig.2. EFS estimation accuracies achieved with WLRS and LSR in site 1 for $\mathrm{N}=60$
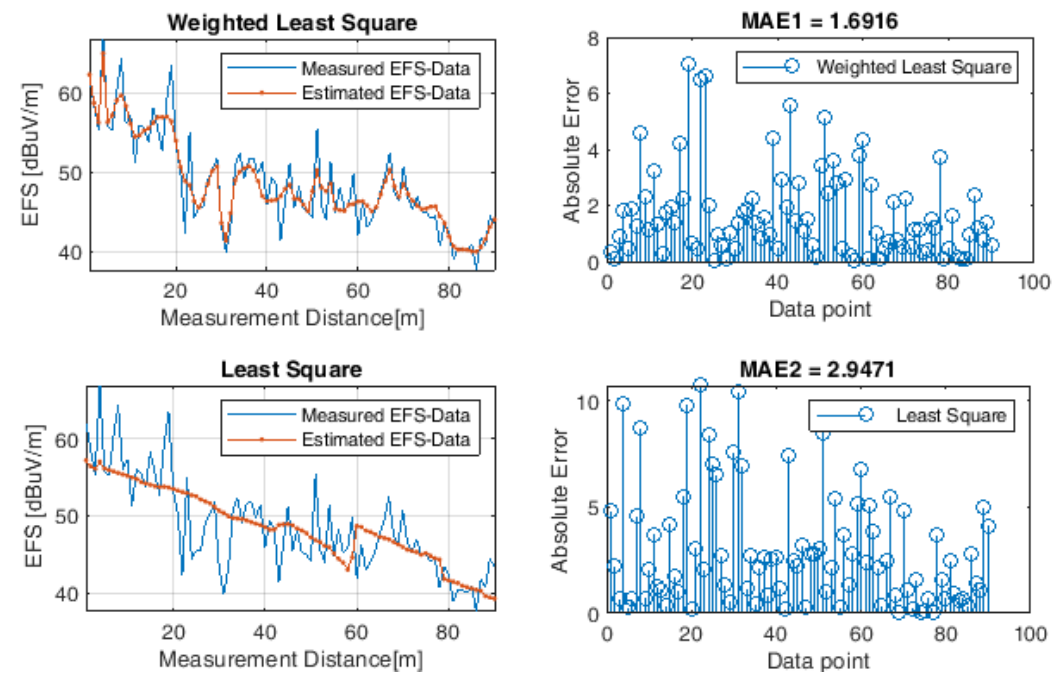

Fig.3. EFS estimation accuracies achieved with WLRS and LSR in site 1 for $\mathrm{N}=90$ 

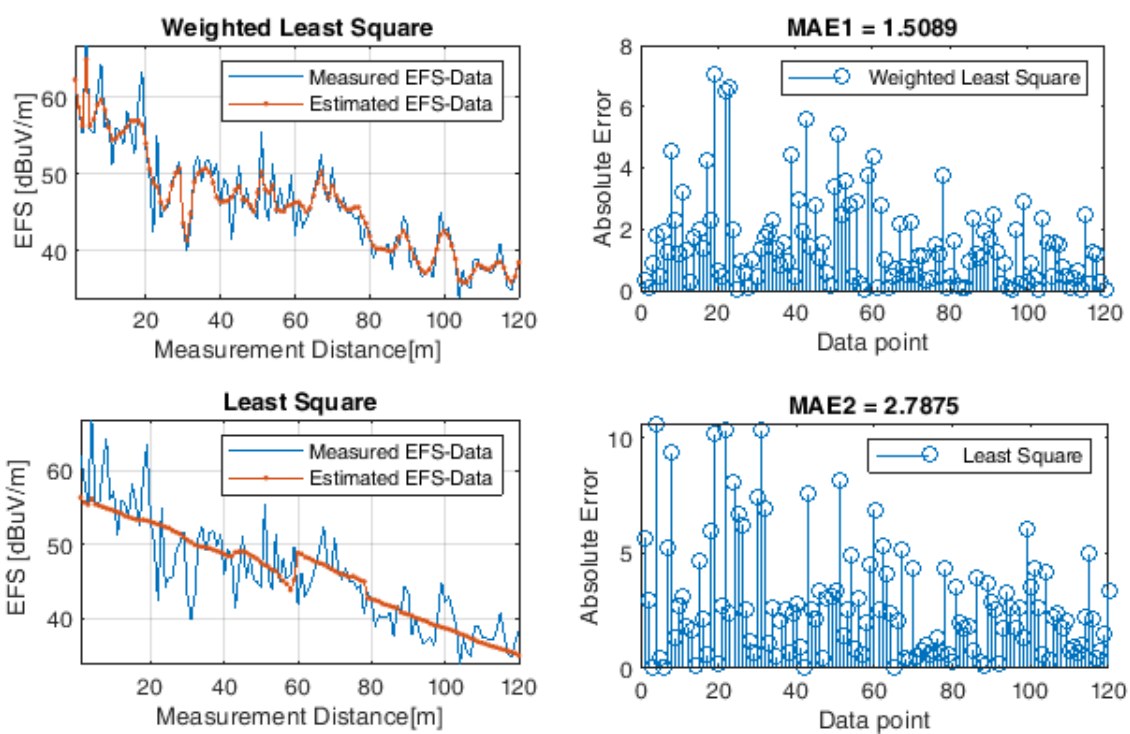

Fig.4. EFS estimation accuracies achieved with WLRS and LSR in site 1 for $\mathrm{N}=120$
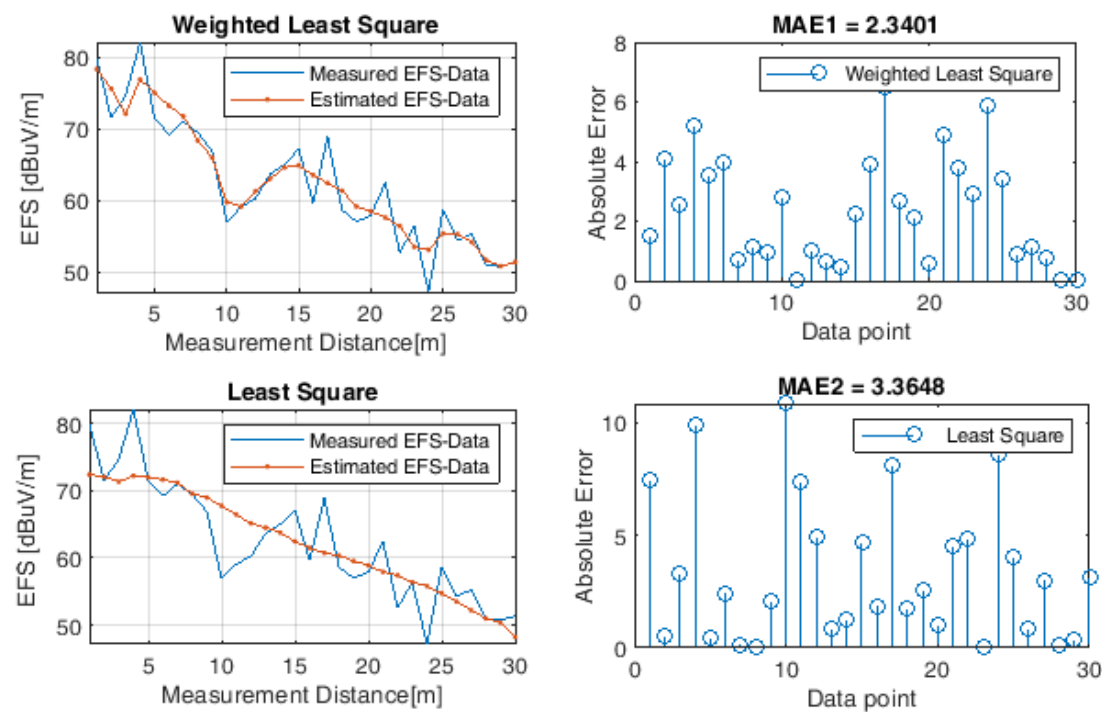

Fig.5. EFS estimation accuracies achieved with WLRS and LSR in site 2 for $\mathrm{N}=30$
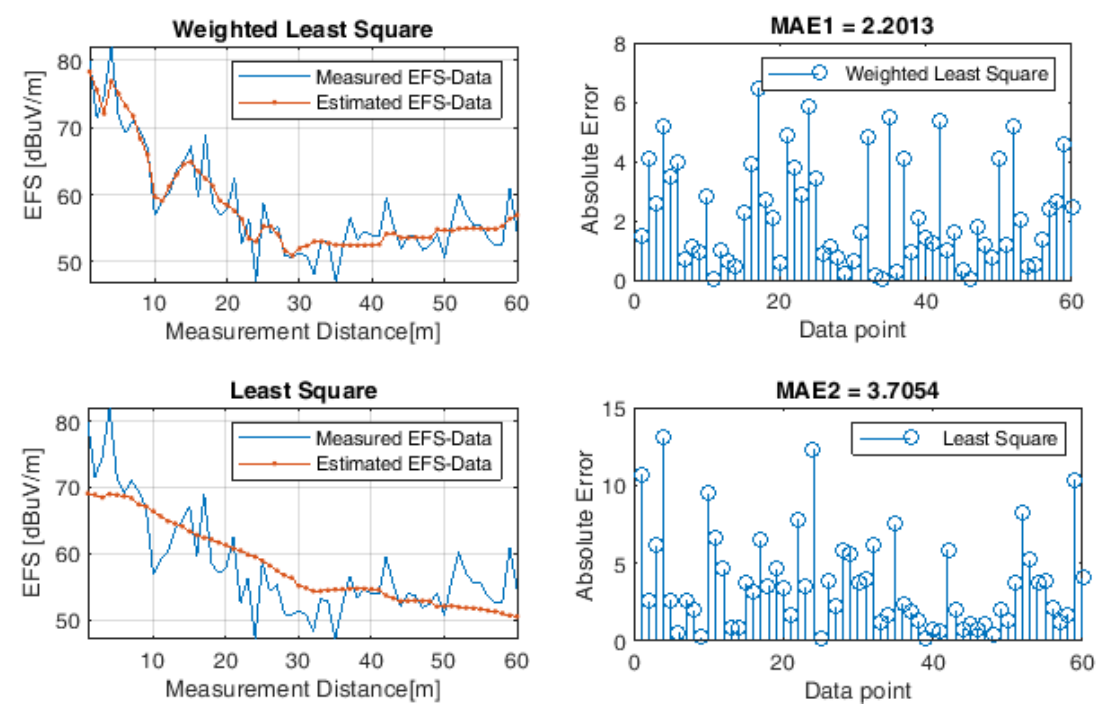

Fig.6. EFS estimation accuracies achieved with WLRS and LSR in site I for N=60 

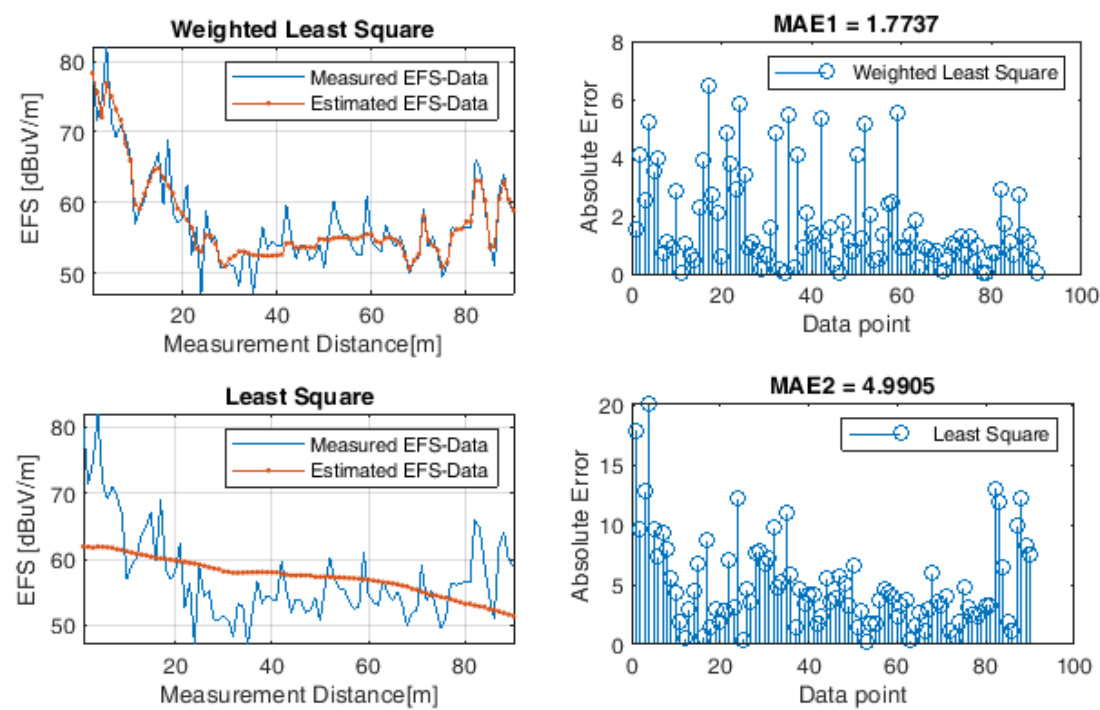

Fig.7. EFS estimation accuracies achieved with WLRS and LSR in site II for $\mathrm{N}=90$
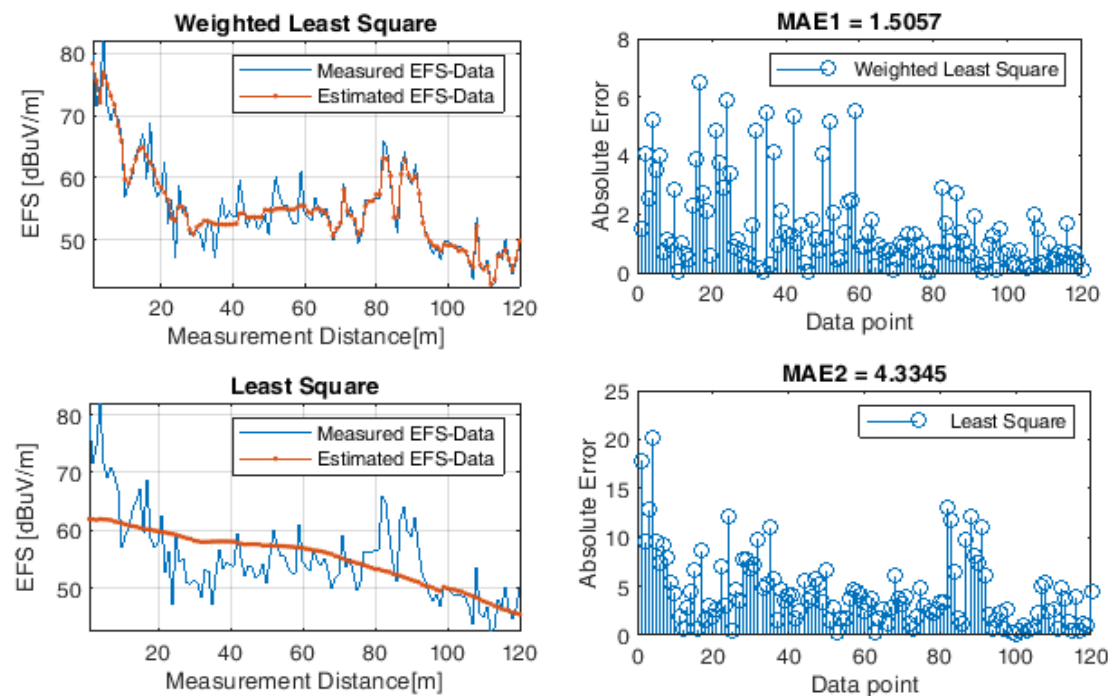

Fig.8. EFS estimation accuracies achieved with WLRS and LSR in site II for $\mathrm{N}=120$

Table 1. EFS estimation accuracies achieved with WLRS and LSR for N=30, 60, 90 and 120 eNodeB sites I and II

\begin{tabular}{|c|c|c|c|c|c|c|c|c|}
\hline & \multicolumn{4}{|c|}{ Weighted Least Square } & \multicolumn{4}{|c|}{ Last Square } \\
\hline & $\mathrm{N}=30$ & $\mathrm{~N}=60$ & $\mathrm{~N}=90$ & $\mathrm{~N}=120$ & $\mathrm{~N}=30$ & $\mathrm{~N}=60$ & $\mathrm{~N}=90$ & $\mathrm{~N}=120$ \\
\hline Lo & 63.23 & 58.60 & 57.92 & 56.90 & 175.43 & 70.27 & -77.86 & -78.26 \\
\hline L1 & -0.05 & -0.02 & -0.02 & -0.01 & -0.38 & -0.05 & 0.13 & 0.10 \\
\hline MAE & 3.27 & 3.48 & 2.99 & 2.78 & 1.90 & 2.24 & 1.99 & 1.79 \\
\hline RMSE & 4.17 & 4.53 & 3.99 & 3.72 & 2.73 & 2.88 & 2.60 & 1.38 \\
\hline PE & 19.50 & 20.55 & 20.60 & 20.61 & 13.72 & 14.18 & 14.14 & 14.18 \\
\hline STD & 2.59 & 2.90 & 2.69 & 2.47 & 1.95 & 1.78 & 1.67 & 1.56 \\
\hline $\mathrm{R}^{2}$ & 0.5317 & 0.4431 & 0.6844 & 0.7258 & 0.8494 & 0.7890 & 0.8262 & 0.8890 \\
\hline Lo & 74.63 & 70.39 & 62.28 & 62.31 & 43.64 & 24.60 & 131.60 & -266.72 \\
\hline L1 & -0.08 & -0.04 & -0.01 & -0.01 & 0.02 & 0.07 & -0.08 & 0.23 \\
\hline MAE & 3.36 & 3.70 & 4.99 & 4.33 & 2.66 & 2.41 & 2.06 & 1.82 \\
\hline RMSE & 4.57 & 4.81 & 6.25 & 5.66 & 4.24 & 3.01 & 2.65 & 2.40 \\
\hline $\mathrm{PE}$ & 15.99 & 20.70 & 32.55 & 43.49 & 12.60 & 12.60 & 12.60 & 12.60 \\
\hline STD & 3.09 & 3.09 & 3.77 & 3.65 & 1.85 & 1.79 & 1.67 & 1.56 \\
\hline $\mathrm{R}^{2}$ & 0.7232 & 0.6122 & 0.1642 & 0.3341 & 0.8611 & 0.8484 & 0.8496 & 0.8923 \\
\hline
\end{tabular}




\section{Conclusion}

Regression measures and techniques are often utilized to model measurement variables and estimate distributional parameters owing of their distinctive computational characteristics and simplified application framework. A wellknown and dominant field strength modelling and estimation technique is literature is the least square regression (LSR). However, LSR is known to be very sensitive to extreme observations (i.e. outliers) and unusual stochastic observations. Also, for many non-Gaussian situations, the LSR is far fetch from providing optimal solutions, particularly when the errors trail longer tails type of distributions

In this paper, firstly, we have shown the levels of measured field signal variations over LTE cellular network interface, using two eNodeB sites as case study. More importantly, we have explore and apply the proposed robust adaptive weighted regression technique over the commonly used LSR method for enhanced propagation practical field strength estimation modelling over cellular radio communication networks interface. We found that the proposed weighted least square regression modeling technique recorded a better field strength data estimation performance compared the standard LSR approach. Such excellent performance of the proposed robust regression technique can be attributed to its weighted factor which enable to cater for any form of data sample sizes of kinds and usual data values. Such robust field strength estimation-based modelling scheme is recommended for proper modeling design, simulation and analysis of present and future communication systems.

\section{References}

[1] Isabona, J, and Srivastava, V.M. (2017), Radio Channel Propagation Characterization and Link Reliability Estimation in Shadowed Suburban Macrocells, International Journal on Communications Antenna and Propagation (IRECAP), Vol. 7 (1), pp. 57-63.

[2] Isabona, J, and Ojuh, D. O. (2017) Wavelet Selection Based on Wavelet Transform for optimum Noisy Signal Processing, International Journal of Basic and Applied Sciences, Vol. 3, Issue 1, pp. 57-65 OFaculty of Basic and Applied Sciences, Benson Idahosa University, Benin City, Nigeria.

[3] C. K. Mai, I. V. M. Krishna, and A. V. Reddy, "Polyanalyst application for forest data mining," in Proceedings of IEEE International Geoscience and Remote Sensing Symposium (IGARSS '05), vol. 2, pp. 756-759, July 2005.

[4] Y. Zhou, S. C. Huang, and M. Bergsneider, "Linear ridge regression with spatial constraint for generation of parametric images in dynamic positron emission tomography studies," IEEE Transactions on Nuclear Science, vol. 48, no. 1 I, pp. 125-130, 2001.

[5] Y. Zhou, S. C. Huang, and Y. I. Hser, "Generalized ridge regression versus simple ridge regression for generation of kinetic parametric images in PET," in Proceedings of IEEE Nuclear Science Symposium, vol. 3, pp. 1551-1555, 1999.

[6] C. K. Mai, I. V. M. Krishna, and A. V. Reddy, "Polyanalyst application for forest data mining," in Proceedings of IEEE International Geoscience and Remote Sensing Symposium (IGARSS '05), vol. 2, pp. 756-759, July 2005.

[7] G. Sebastiani, F. Godtliebsen, R. A. Jones, O. Haraldseth, T. B. M"uller, and P. A. Rinck, "Analysis of dynamic magnetic resonance images," IEEE Transactions on Medical Imaging, vol. 15, no. 3, pp. 268-277, 1996.

[8] K. Diawuo,K., Dotche, K.A and T Cumberbatch, "Data Fitting to Propagation Model Using Least Square Algorithm: A Case Study in Ghana", International Journal of Engineering Sciences, 2(6), 226-230, 2013.

[9] J. Isabona, and S.O. Azi. S.O "Optimised Walficsh-Bertoni Model for Pathloss Prediction in Urban Propagation Environment", International Journal of Engineering and Innovative Technology (IJEIT) Vol. 2, Issue 5, pp 14-20, 2012.

[10] J.Isabona and C.C. Konyeha. "Experimental Study of UMTS Radio Signal Propagation Characteristics by Field Measurement", American Journal of Engineering Research, vol. 2, (7), pp 99-106.

[11] J. Isabona and C.C. Konyeha. C.C "Urban Area Path loss Propagation Prediction and Optimisation Using Hata Model at 800MHz”, IOSR Journal of Applied Physics (IOSR-JAP), Vol. 3, Issue 4, pp.8-18, 2013.

[12] J. Isabona and G.P. Isaiah. "CDMA2000 Radio Measurements at $1.9 \mathrm{GHz}$ and Comparison of Propagation Models in Three Built-Up Cities of South-South, Nigeria", American Journal of Engineering Research (AJER), Vol. 2, Issue-05, pp-96-106, 2013.

[13] J. Isabona, and S.O. Azi. S.O "Enhanced Radio Signal Loss Prediction with Correction Factors for Urban Streets in the IMT-2000 Band", Elixir Space Science, vol. pp.15958-15962, 2013.

[14] J. Isabona, and M. Babalola, "Statistical Tuning of Walfisch-Bertoni Pathloss Model based on Building and Street Geometry Parameters in Built-up Terrains". American Journal of Physics and Applications, vol. 1, pp. 10-16, 2013.

[15] J. Isabona, C.C. Konyeha, C.B. Chinule and G.P. Isaiah "Radio Field Strength Propagation Data and Pathloss calculation Methods in UMTS Network", Advances in Physics Theories and Applications, vol.21.pp 54-68, 2013.

[16] J. Isabona,.G. P. Isaiah "Computation and Verification of Propagation Loss Models based on Electric Field Data in Mobile Cellular Networks, Australian Journal of Basic and Applied Sciences, Vol. 9 (29), pp 280-285, 2015. 
[17] Isabona Joseph, Divine O. Ojuh, " Adaptation of Propagation Model Parameters toward Efficient Cellular Network Planning using Robust LAD Algorithm", International Journal of Wireless and Microwave Technologies(IJWMT), Vol.10, No.5, pp. 13-24, 2020.DOI: 10.5815/ijwmt.2020.05.02

[18] M.K Yeliz, "Generalized Least Square and Weighted Least Squares Estimation Methods for Distributional Parameters', REVSTAT - Statistical Journal, Vol. 13 (3), pp.263-282, November 2015.

[19] F. H. Thanoon. "Robust Regression by Least Absolute Deviations Method" International Journal of Statistics and Applications, 5(3): 109-112, 2015. DOI: 10.5923/j.statistics.20150503.02

[20] Y.M Kantar, Generalized Least Squares and Weighted Least Squares Estimation Methods for Distributional Parameters, REVSTAT - Statistical Journal Vol. 13 (3), 263-282, 2014.

[21] J. Isabona, “ Wavelet Generalized Regression Neural Network Approach for Robust Field Strength Prediction', Wireless Personal Communication (Springer) https://doi.org/10.1007/s11277-020-07550-5, 2020.

[22] I.B. Oluwafemi, and O. J. Femi-Jemilohun. "Propagation Profile and Strength Variation of VHF Signal in Ekiti State Nigeria', .J. Wireless and Microwave Technologies, Vol 7, No.3, pp. 9-24, 2017.

[23] Akinsanmi Akinbolati, Olufemi J. Agunbiade, " Assessment of Error Bounds for Path Loss Prediction Models for TV White Space Usage in Ekiti State, Nigeria", International Journal of Information Engineering and Electronic Business(IJIEEB), Vol.12, No.3, pp. 28-39, 2020. DOI: 10.5815/ijieeb.2020.03.04

\section{Authors' Profiles}

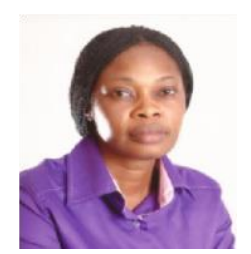

Dr. (Mrs.) O. D. Ojuh is an academic staff of Benson Idahosa University, Benin City, where she lectures Physics. She obtained her Ph.D. and M.Sc in Theoretical/ Mathematical Physics at the University of Benin City, Nigeria in 2012 and 2007 respectively and a B.Sc in Physics in 1996, from then Edo State University now known as Ambrose Alli University, Ekpoma, Edo State. Her research interest are Computational condensed matter physics/materials Science for renewable energy applications and Physics of radio signal propagation engineering. She can be reached through Email dojuh@ biu.edu.ng.

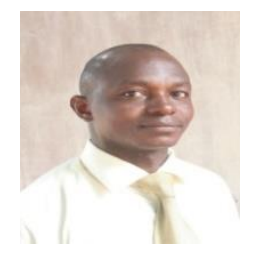

Joseph Isabona, Ph.D, received Ph.D. and M.Sc. degrees in Communication Electronics, 2013 and 2007 respectively, and a B.Sc in Applied Physics in 2003. He is the author of more than 100 scientific contributions including articles in international refereed Journals and Conferences in the area of Wireless Mobile communications. The Author is a Postdoctoral Research Fellow of the Department of Electronic Engineering, Howard College, University of KwaZulu-Natal, Durban, South Africa. His area of interest includes Signal Processing, Radio Resource Management and Physics of radio signal propagation engineering. She can be reached with josabone@yahoo.com.

How to cite this paper: Divine O. Ojuh, Joseph Isabona, "Field Electromagnetic Strength Variability Measurement and Adaptive Prognostic Approximation with Weighed Least Regression Approach in the Ultra-high Radio Frequency Band", International Journal of Intelligent Systems and Applications(IJISA), Vol.13, No.4, pp.14-23, 2021. DOI: 10.5815/ijisa.2021.04.02 